\title{
Optimisation of gate location based on weld line in plastic injection moulding using computer-aided engineering, artificial neural network, and genetic algorithm
}

\author{
R. Sedighi ${ }^{1}$, M.Saleh Meiabadii ${ }^{2 *}$ and M. Sedighi ${ }^{3}$ \\ ${ }^{1}$ Department of Mechanical Engineering \\ Islamic Azad University Karaj Branch, Karaj, Iran \\ ${ }^{2}$ Department of Industrial Engineering, \\ Islamic Azad University, Malayer Branch, Malayer, Iran \\ ${ }^{3}$ School of Environment, Science and Engineering, \\ Southern Cross University, Australia \\ *E-mail: saleh.meiabadi@malayeriau.ac.ir
}

\begin{abstract}
Gate location is one of the most important design elements of an injection mould. Injection moulding is a very complex process with several parameters having interactive effects on each other; hence, computer-aided engineering and artificial intelligence were utilised to optimise mould design based on weld line. Therefore, the finite element analysis, artificial neural network and genetic algorithm were linked to find optimum gate location in a plastic product. A reliable numerical model was developed by Moldflow software based on a real product to simulate injection process. Moldflow was used to predict weld line length and position of the part. Weld lines are visually undesirable and a plastic part is structurally weak at weld line positions. This study describes how the weld line was formed on the part. Polyamide-6 (PA-66) was used as the plastic material. To find optimum gate location, the finite element predictions were implemented to train a neural network, which was used later in the genetic algorithm. The optimisation objective was to find gate location which led to minimum weld line length. This research concluded that the developed procedure can efficiently optimise complex manufacturing processes and prevent flaws in products and thus, can be applied practically in the injection moulding process.
\end{abstract}

Keywords: Optimisation; gate location; weld line; computer-aided engineering; neural network; genetic algorithm

\section{INTRODUCTION}

Injection moulding process is one of the most widely used manufacturing processes employed for the fabrication of plastic products. Although this process is very complicated, its benefits include minimal losses from scrap and finishing requirements result in having a remarkable portion of the plastic production in the world. The quality of plastic injection moulded parts is the result of interaction between several parameters including material properties, geometry part, the design of the injection mould, and processing parameters $[1,2]$. Plastic and machine are usually factors determined by manufacturers and consumers. Therefore, the design of the mould and processing 
parameters are the controlling factors to obtain the desired features in the injected products.

Design and process optimisation can be employed in the injection moulding to determine mould geometry and processing parameters so as to meet the manufacturing objectives. Several studies have been conducted to determine the impact of the process parameters (e.g., injection velocity, mould temperature, melt temperature, gate dimension, filling pressure and time, cooling time and injection time) on product qualities and defects (e.g., strength, warpage, and shrinkage) [3-10]. Injection mould design and process parameters determined using a trial-and-error fine-tuning based on past experience are time and cost consuming and offer no guarantee of an optimal solution. In the past three decades, computer-aided engineering (CAE) has been developed for the prediction of the high-quality injection moulded parts before physically fabricating a mould or part. However, CAE requires the mould designer to run several simulations and evaluate the results and subsequent adjustment based on the results until satisfactory results are obtained. This manual adjustment process also does not guarantee an optimal solution. Thus, there is an increasing interest in the utilisation of efficient techniques for optimising plastic injection moulding. Artificial neural networks were used in many manufacturing processes for modelling the processes [11-15] and a genetic algorithm was also used to optimise lots of manufacturing processes [16-18].

According to the literature, a hybrid system of computer-aided engineering and artificial intelligence was utilised by Meiabadi et al. [1] to optimise plastic injection moulding process parameters to enhance the quality of a moulded part and decrease process cycle time. Spina [19] introduced the evaluation of the finite element (FE) simulation results through the ANN system. Results demonstrated by ANN system are an efficient method to assess the influence of variation of process parameters on part manufacturability and improve part quality. Kurtaran et al. [20] applied finite element software, Moldflow and linked to ANN in order to find the optimum conditions of injection moulding process for a bus ceiling lamp. As a result, the maximum warpage of the initial model decreased by $46.5 \%$. Artificial neural network and genetic algorithm were used together by Changyu et al. [21]. The results clearly showed that the quality index of the volumetric shrinkage variation on the part was improved. More importantly, Ozcelik et al. [22] confirmed that the combination of artificial neural network and genetic algorithm gave satisfactory results to minimise warpage of thin shell plastic parts in injection moulding process. A VOF method coupled with a finite volume approach was used for simulating mould insert injection moulding process. Tutar and Karakus [23] demonstrated that mass inflow rate acted not only on the evolution of injection pressure but also affected the rate of temperature increase on the outer surface of the metallic insert and solidification percentage and thus, it became the principal parameter for optimising the polymer melt flow. Huang et al. [9] used the Moldflow with the results predicted by a prognostic model proposed by the authors to predict this complicated process. The warpage, shrinkage, and volumetric shrinkage were studied by Chen et al. [24]. They combined the finite element software, MoldFlow with Taguchi method. Chen and Kurniawan [25] applied Taguchi method, BPNN, GA, and hybrid PSO-GA to find optimum parameter settings for multiple quality characteristics. In another research, Spina et al. [26] investigated the lens manufacturing with the injection moulding process by using the commercial software Moldflow insight and grey relational Taguchi-based component to identify the optimal parameter sets. Most of the articles have studied the minimising of defects like shrinkage or warpage by optimising the processing parameters but the purpose of this research innovatively is on optimisation of gate location based on 
minimisation of weld line length. In this study, plastic injection moulding process is modelled by the neural network, a random approach used to create solution space. Based on injection goal, optimised gate location is obtained by genetic algorithm. Finite element software Moldflow, artificial neural network and genetic algorithm are exploited to find optimal gate location. Finally, the efficiency of optimisation method is confirmed by experimental fabrication. The trade name of the plastic is Pa66Akulon NY-8 Natl. Table 1 presents the material properties.

Table 1. Material properties.

\begin{tabular}{ll}
\hline Parameter & Value and unit \\
\hline Melt Density & $0.9049 \mathrm{~g} / \mathrm{cm}^{3}$ \\
Solid Density & $1.0891 \mathrm{~g} / \mathrm{cm}^{3}$ \\
Shear modulus & $640 \mathrm{MPa}$ \\
Elastic modulus & $2034 \mathrm{MPa}$ \\
\hline
\end{tabular}

\section{MODELLING AND OPTIMISATION}

\section{Optimisation Objectives}

Gate is one of the most important design elements of an injection mould and has a large bearing on the quality of the part. Gate location has a large impact on weld line formation and location. Therefore, weld line length is selected as the part quality index to investigate effects of gate location on weld line behaviour. Weld line is always considered as a common issue related to injection moulding process and the main target to be avoided by mould designers [27]. Weld lines are formed when two melt fronts come in contact with each other. Separation or division of the melt flow front can be caused by flow obstructions such as cores and holes, melt front race-tracking due to variable wall thickness, jetting, unfavourable gate location, or runner branching for multi-gated parts. The mechanical properties of weld line infested areas are inferior to the properties of the bulk. The surface of weld-line may contain small cracks which influence appearance of the moulded parts [28]. Modifying the gate location to facilitate the transmission of pressure and maintain a higher melt temperature is in favour of improving weld lines properties [29]. Large gate size and shorter distance between gate and weld line provide a better quality of the weld line [10]. The best condition to avoid weld line is by filling the mould by balancing flow pattern and reaching the same point simultaneously. Therefore, the optimisation objective is to find the best gate location to minimise weld line length.

\section{Optimisation Procedure}

Computer-aided engineering, artificial neural networks and genetic algorithm are employed to determine optimum gate location through a systematic optimisation approach. CAE is used to simulate the manufacturing process. ANN and GA are two of the most influential artificial intelligence tools to incorporate into the optimisation procedure. One of the most attractive features of ANNs is the ability to produce an approximate solution of nonlinearities when the input and output are clear while the explicit relationship information is incomplete [22]. The genetic algorithm is a method for solving optimisation problems based on natural selection; the process that drives biological evolution. Combining ANN model with GA is a promising computation optimisation technique to find optimum gate location. The hybrid system of computer- 
aided engineering and artificial intelligence can be used when multi-response quality characteristics are needed. Figure 1 demonstrates the flowchart combining ANN/GA optimisation.

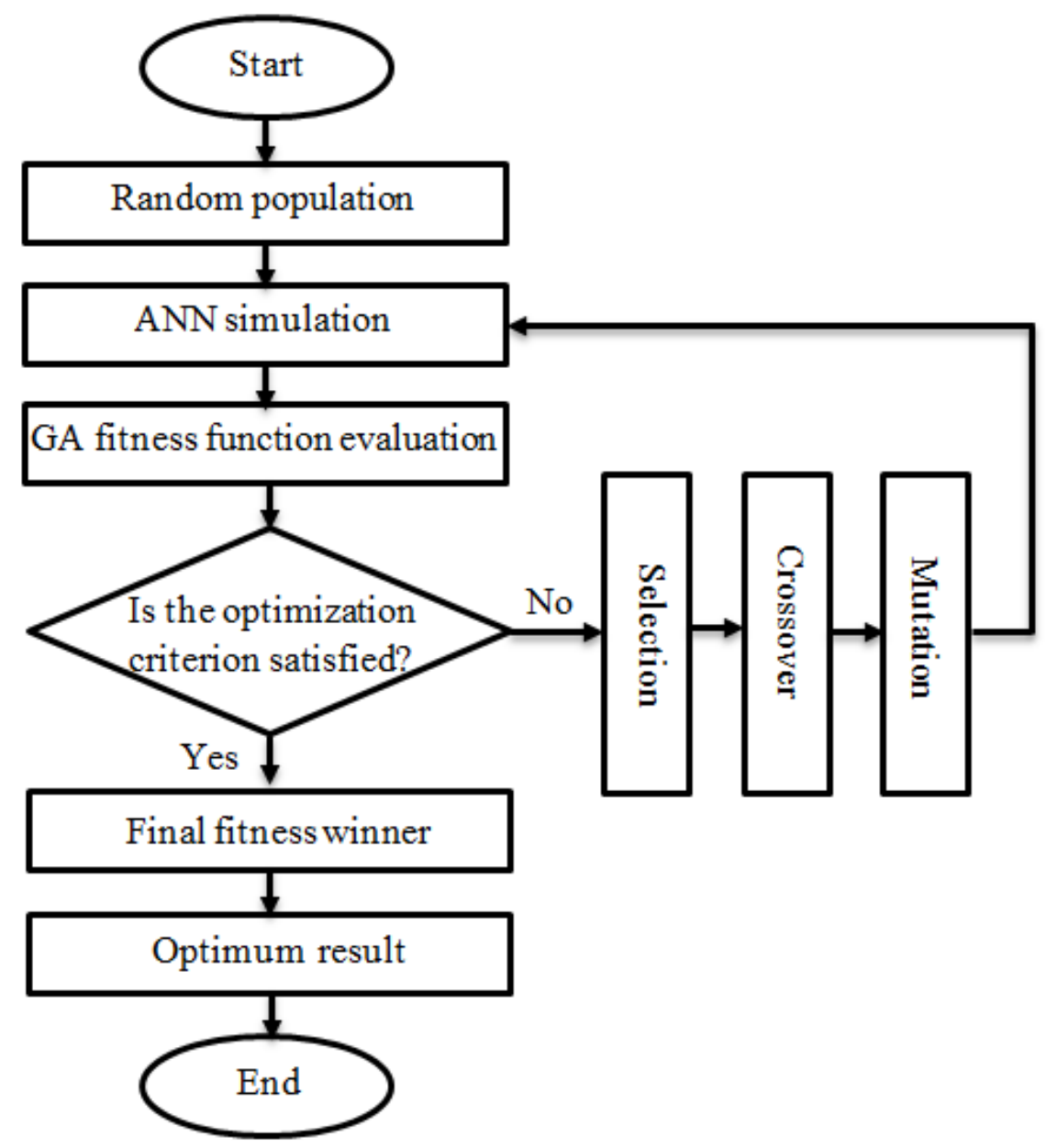

Figure 1. Flowchart of combining ANN/GA optimisation.

\section{Simulation of Injection Process and Validation of the Simulation}

Computer-aided engineering is used to simulate practical experiments due to cost and time saving advantages. Moldflow Plastics Insight (MPI) is one of the commercial CAE software to simulate plastic injection moulding process. Moldflow software represents the most comprehensive capability to evaluate mould design. The CAD model of the product was initially imported and converted into a finite element mesh model. Simulation results of the injection process are used for modelling injection process by neural network. However, simulation results should be confirmed by experimental fabrication of the product. Results show that there is a good agreement between location and length of the simulated weld line and experimental specimen. The weld line is formed at the same location as the weld line of real specimen. Comparison of the simulated specimen with the real product is shown in Figure 2. The matching between simulated and real specimen confirmed the validation of Moldflow simulations. The only difference between the predicted and actual weld line was the upper tip of the weld line. In the real specimen, there are two separate weld lines at the upper side. 

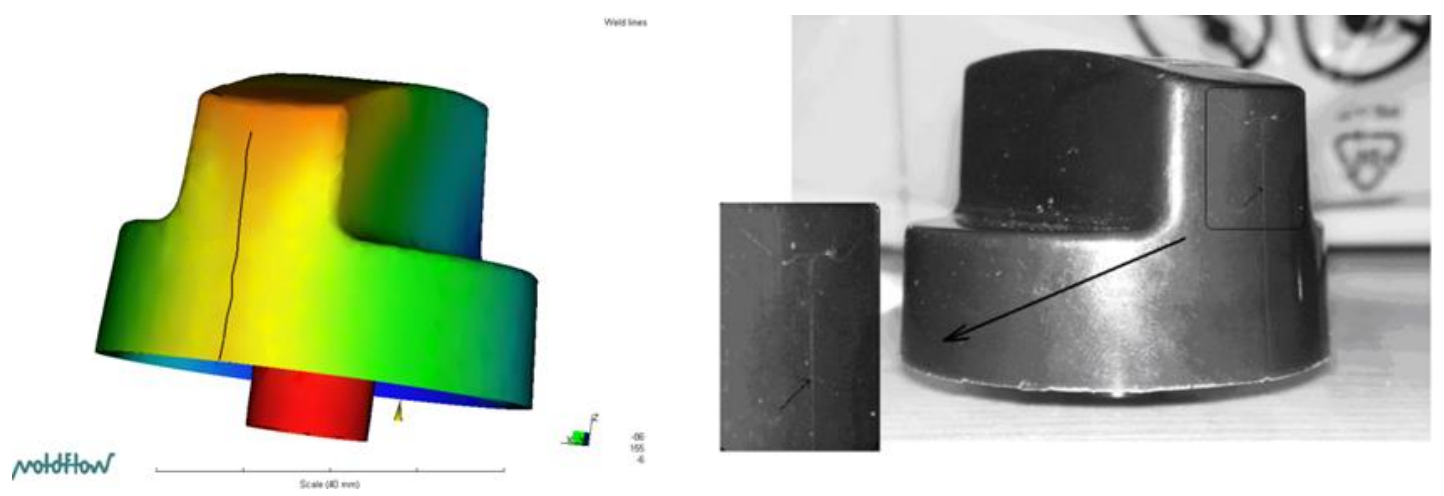

Figure 2. Comparison of the simulated specimen with the real product.

It is believed that this discrepancy between the predicted location of the weld line and actual position is due to the limited number of the FE meshes. In addition, it is assumed in the simulation that the temperature profile of the mould is uniform. This may not be the case if a non-uniform cooling system is being used in the production line. Flow is divided into three fronts which meet each other at the weld line location. There are common reasons causing weld line formations including i) flow obstacles in the mould, ii) the part thickness is variable, and iii) a multi-gating option is adopted. In this study, the geometry shape and obstacles lead to the weld line formation. The geometry is formed from two different parts including a cylinder shape and cube which have different height. When the fluid is injected to the mould, it moves to different paths of rectangle knob and lateral surface of the cylinder. The shape, which has shorter height and path, is filled sooner than the shape which has a longer path. Therefore, the flow path is divided into three fronts. Pressure gradient must be uniform during filling process. Figure 3 represents the pressure at injection point which is uniform. A sharp increasing at the beginning of analysis was predictable. While pressure gradient is uniform there is an unbalance flow pattern in the specimen (Figure 4(c)).

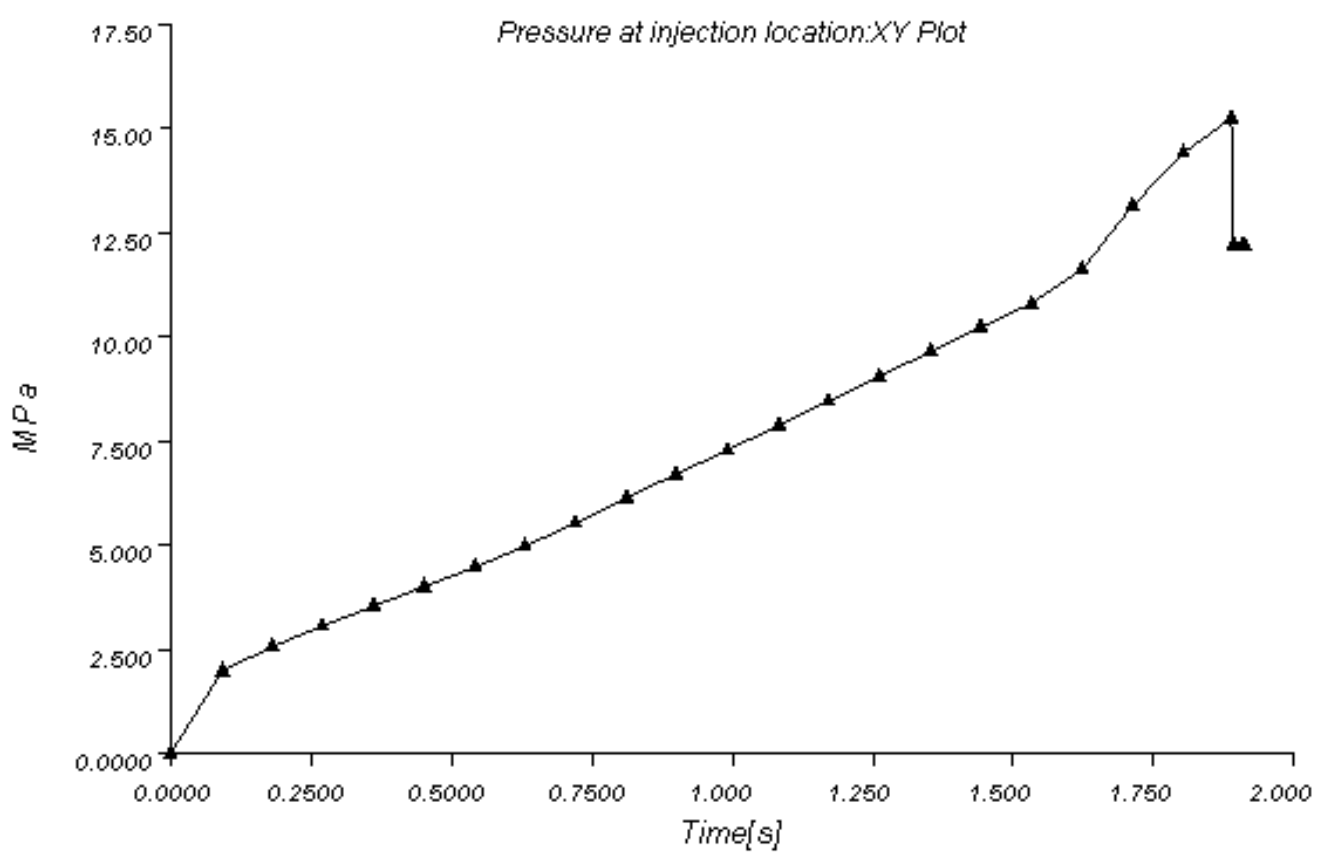

Figure 3. Time variation of pressure at injection location. 
The mould is filled based on radius pattern. As the flow pattern was not uniform (see Figure 4(c)), extra material was compressed into one flow path (weld line position) while another flow path (small cylinder base) was still being filled. This phenomenon is named over-packing. The shortest and thickest flow paths were filled first, resulting in over-packing which caused several flaws including excessive cycle time, part weight, warpage and flashing and generally occurred in sections with the shortest fill time. During small cylindrical base filling, the pressure line became a little sharper. Finally, by filling the cylindrical base, the filling process was completed so the pressure suddenly decreased as shown in Figure 3. Figure 4 illustrates the different parameters including filling time, flow orientation, frozen layer, the temperature of flow front, and bulk temperature at the end of filling. Moldflow is able to predict the molecular orientation at the skin (Figure 4(b)) as well as at the core of the specimen (Figure 4(a)). There are significant changes in polymer flow orientation, shear rate, and shear stress during filling of the mould. When the mould is being filled, the hot polymer is stuck to cold mould so it makes the frozen layer. These frozen layers increased the flow resistance. Then the polymer flowed in the mould cavity as fountain flow. Increasing the velocity gradient increased shear stress. Therefore, significant shear stress occurred between molecules and consequently the molecules were drawn and aligned with the flow direction. This is the main reason for the differences of molecule orientation between skin and core of specimen as shown in Figure 4(a-b). In addition, the differences in molecular orientation are some of the major reasons for the weakness of the weld line.The frozen layer fraction result generated from flow analysis showed the thickness of the frozen layer as a fraction. A thicker frozen layer corresponded to a higher value as shown in Figure 4(d). Recombining molecular chains led to higher shear stress in weld line position. Consequently, the temperature increased in this area so the frozen layer thickness decreased. This is well illustrated in Figure 4(d) by a vertical line which coincides with the location of the weld line. This also conceded the consistency of the simulation results with the experimental model.

As observed in Figure 4(e), the temperature at flow front decreased with time, thus with regard to the temperature contour, the filling time (transposition) of different parts of the model can be distinguished. Figure 4(e) revealed that during the formation of the weld line, the temperature of flow fronts was low and decreased the strength of the weld line. When the two advancing flow fronts faced each other, the shear stress was developed at the contact point. Therefore, the increase in shear stress increased the temperature in the weld line position as shown in Figure 4(f) (yellow line). This figure reconfirmed the agreement of numerical calculation with the real product. Velocity vectors and field line of time must be perpendicular. As can be seen in Figure 4(b), there was a change in perpendicular arrows' orientation to tangent with respect to the field line around the weld line area. This means that at the beginning of the process, the molecules are perpendicular to the field line; however, after a while and during the filling process, they are oriented to the new directions. This phenomenon is called under flow, which causes poor part quality in both appearance and structural aspects because there is enough time in order to a frozen layer to be developed when two flow fronts meet each other in two opposite directions. Then the polymer in one of the flows reverses direction and as a result, the frozen layer partly re-melted due to frictional heating. 


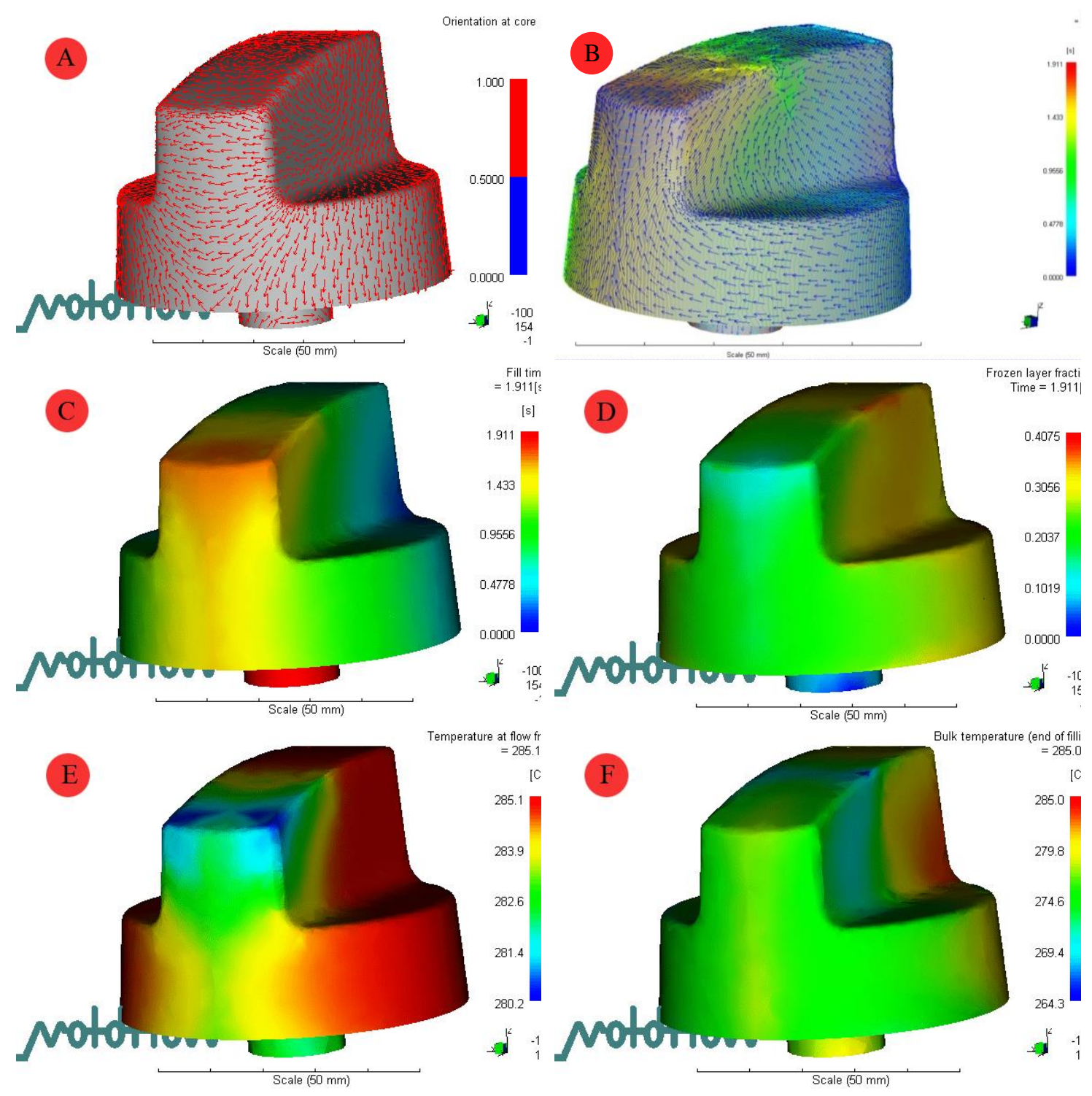

Figure 4. Moldflow analysis results (a) orientation of flow at core (inner surface) of specimen, (b) orientation of flow at skin (visual surface) of specimen and field lines of time, (c) filling time, (d) frozen layer fraction, (e) temperature of flow front, and (f) bulk temperature at the end of filling.

\section{Creating Solution Space}

The coordinates of gate locations are considered as control variables while the quality index is accounted as response variable that can be obtained by simulation of the injection process. Sets of the control variables are called solution space. In this study, solution space is defined randomly. The part is projected from bottom view on two dimensional surface and gate locations randomly distributed on the projected surface. Because of the symmetry, half of the projected surface can be considered as the search area to reduce the optimisation processing time. The centre of projected surface was determined as an origin of the coordinate system. The solution space consisted of 102 points. Simulation of injection process was done by MPI to measure the weld line length on each of the selected points. Figure 5 shows the distribution of the gate locations. 

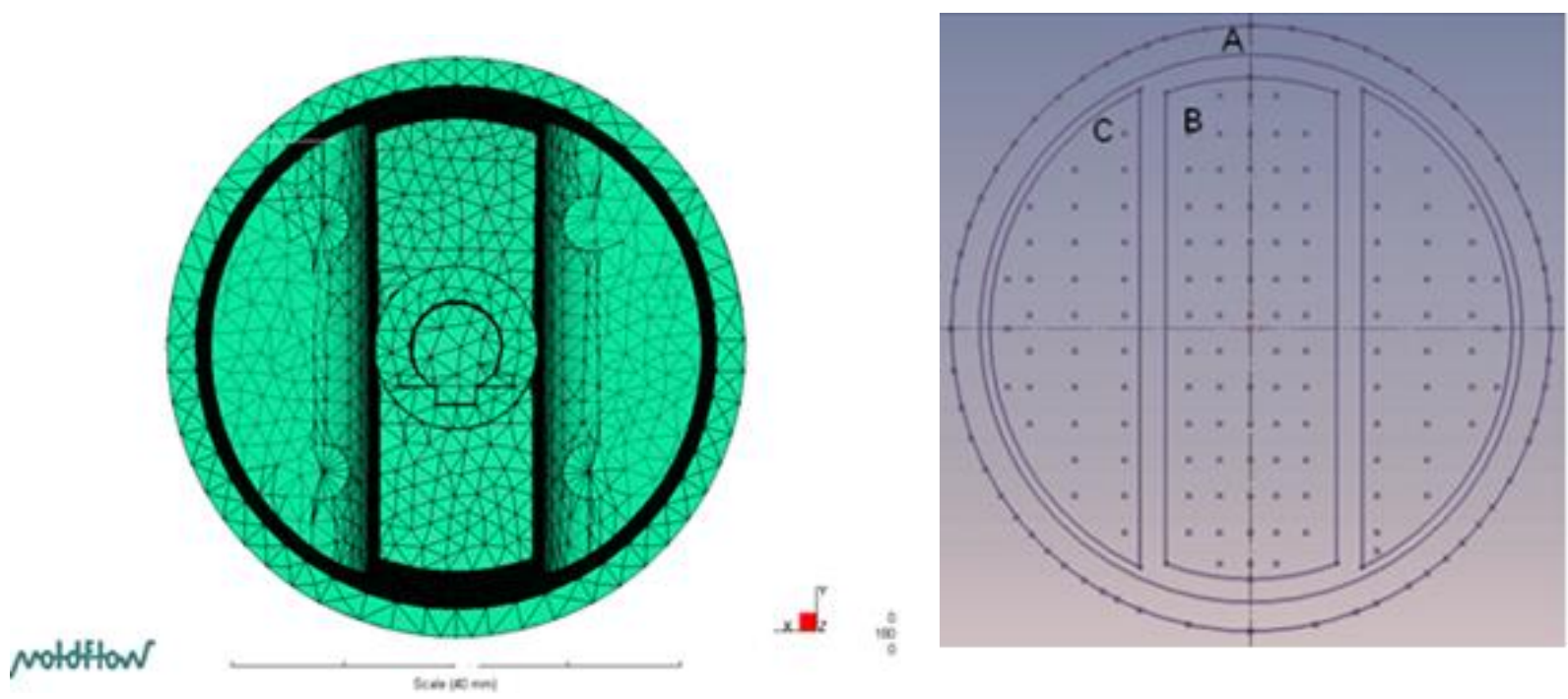

Figure. 5 Distribution of the gate locations.

\section{Modelling Plastic Injection Moulding Process by Neural Network}

The complex relationships between control variables and response variables could not be determined by any analytical model. The neural networks have been shown to be an effective technique for modelling complex nonlinear processes. They are useful for functional prediction and system modelling where the processes are not understood or are highly complex. In this study, a radial basis function (RBF) network, which is an artificial neural network using radial basis functions as activation functions was used. Radial basis networks can require more neurons than standard feed forward back propagation networks but often they can be designed in a fraction of the time it takes to train standard feed forward networks. They work best when many training vectors are available. A radial basis network is a network with two layers; a hidden layer of radial basis neurons and an output layer of linear neurons. The weights and biases of each neuron in the hidden layer define the position and width of a radial basis function. Each linear output neuron forms a weighted sum of these radial basis functions. Each function can be fitted by a radial basis network when the correct weight and bias values for each layer and enough hidden neurons are considered. The radial basis network is trained to respond to specific inputs with target outputs. The dataset used in this study consisted of 102 sets. The trained neural network is capable of predicting weld line length while control variables are applied to the ANN so the suitability of each gate location can be identified.

\section{Finding Optimum Gate Location by Genetic Algorithm}

Optimum coordinates of the gate location were efficiently obtained by genetic algorithm. The genetic algorithm is a method for solving optimisation problems based on natural selection deriving biological evolution. The genetic algorithm repeatedly modifies a population of individual solutions. At each step, the genetic algorithm selects individuals at random from the current population to be parents and uses them to produce children for the next generation. Over successive generations, the population "evolves" towards an optimal solution [26]. To solve the above optimisation problem, GA was coupled with the neural network. It should be mentioned that the direct link between the simulation software and GA required a long time before optimum process parameters were identified [22]. An initial population was generated at random, then, a new generation was 
reproduced by selection; crossover and mutation. The process was repeated until the maximum generation number or population convergence occurred. MATLAB was employed to optimise the injection moulding process according to the above algorithm. In the present research, the population size and value of Elite count were considered 20 and two, respectively. The number of individuals with the best fitness value in the current generation guaranteed to survive to the next generation is called Elite children. The value of crossover fraction was 0.8 . The crossover fraction specifies the fraction of each population, other than the elite children that are made up of crossover children. Figure 6 shows the best fitness achieved after 101 generations. Table 2 demonstrates coordinates of the optimised gate location which was in B area. Figure 7 depicts the location of optimum gate location on the bottom view of the part.

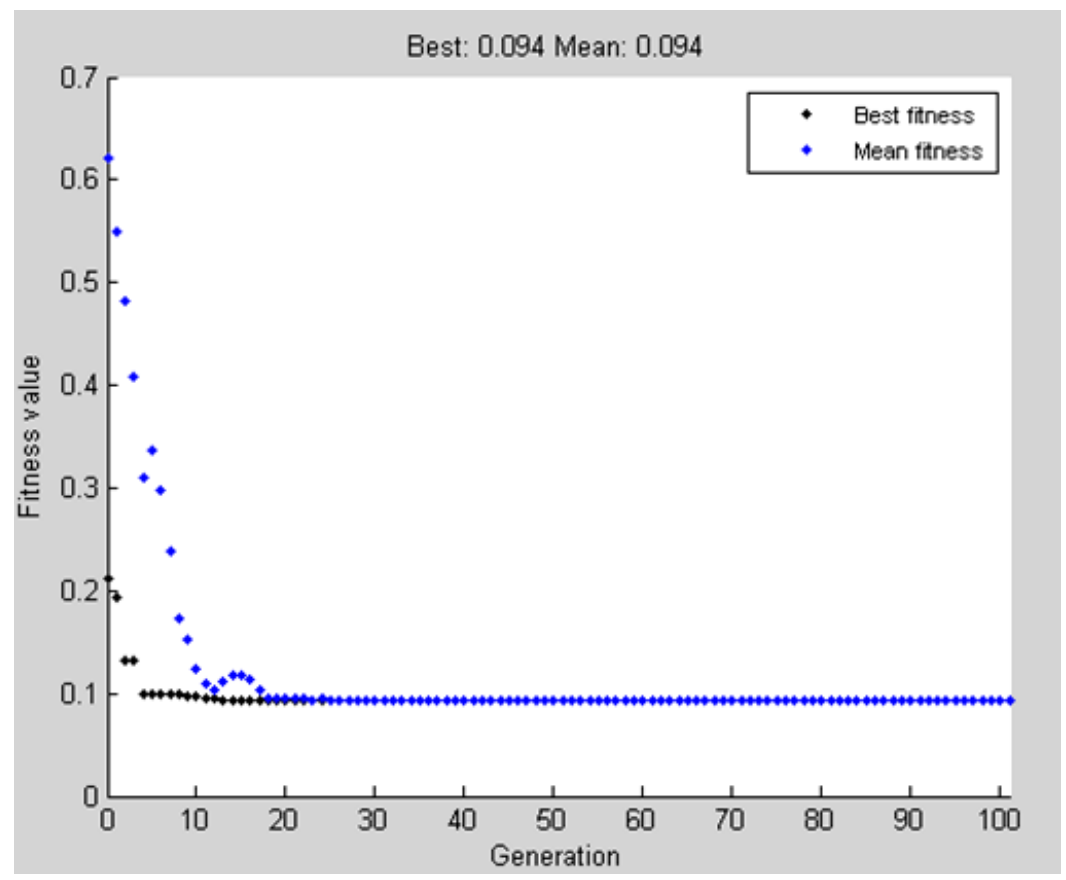

Figure 6. Evolution of generations for injection moulding optimisation.

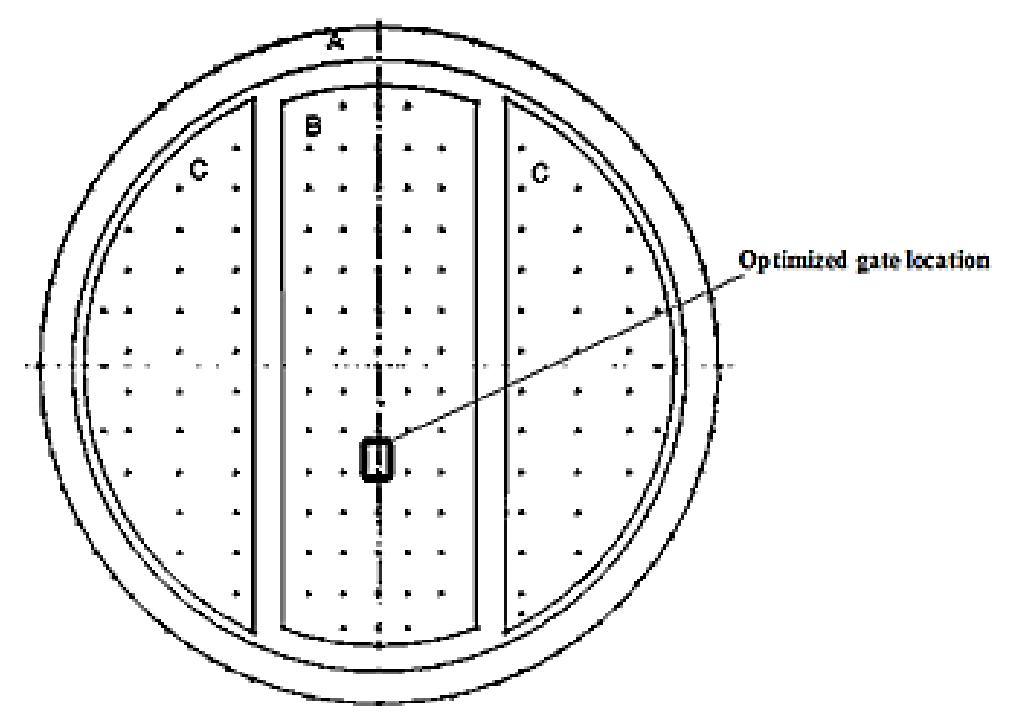

Figure 7. Optimised gate location at bottom view. 
Table 2. Optimised gate location.

\begin{tabular}{cc}
\hline X coordinate of gate & Y coordinate of gate \\
\hline 0 & -19.43 \\
\hline
\end{tabular}

\section{RESULTS AND DISCUSSION}

The optimised gate location was evaluated numerically and experimentally and results showed that there was a good agreement between the experimental and numerical results. Figure 8 a shows that the weld line was removed from the part surface. The comparison between flow pattern before and after gate optimisation in Figure $8 \mathrm{~b}$ and $8 \mathrm{c}$ revealed that the mould was filled by the balanced flow pattern and reached the same point simultaneously so the weld line was removed in the optimised design. Table 3 shows the comparison between results of the Moldflow simulation, neural network, and experimental fabrication using optimal gate location. The results of experimental fabrication were in good agreement with Moldflow and neural network results that revealed the accuracy of Moldflow simulation and also the neural network.

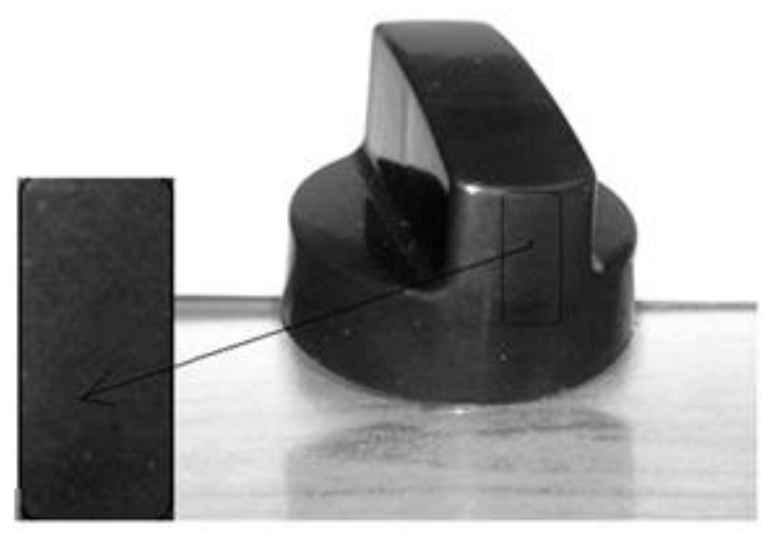

(a)

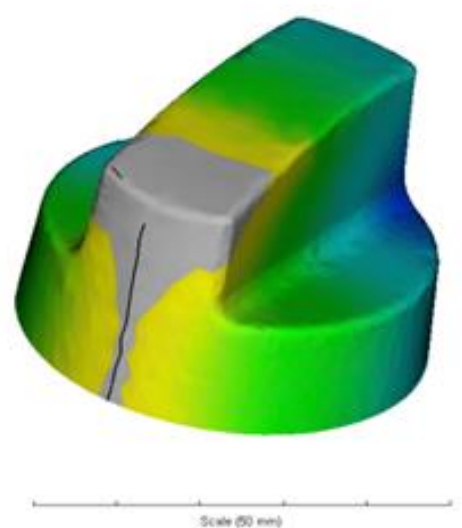

(b)

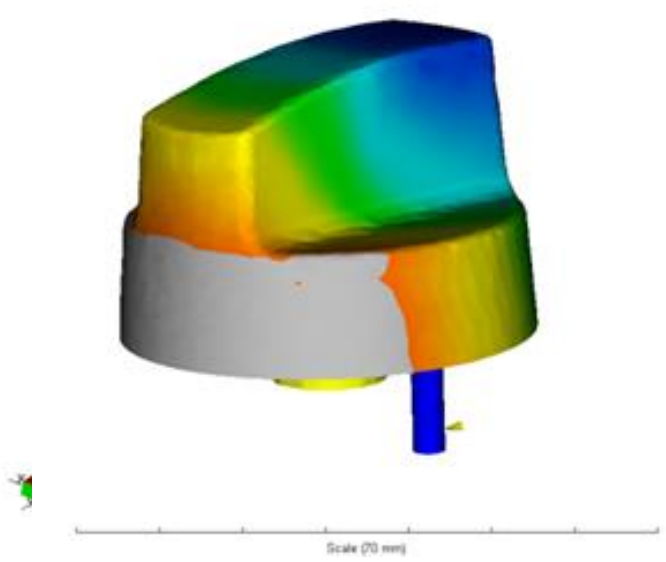

(c)

Figure 8 Experimental fabrication of the product with optimised gate location (a) Comparison between flow patterns, (b) before optimisation, and (c) after optimisation. 
In this research, computer-aided engineering was used to simulate plastic injection moulding process for cost and time-saving purposes. There was a good agreement between finite element analysis and experimental results. Therefore, simulation results were implemented to train a radial basis function (RBF) network. The trained neural network was capable of predicting weld line length while control variables were applied to the ANN. Optimum coordinates of the gate location were efficiently obtained by a genetic algorithm using the optimised gate location results in minimum weld line length which was almost zero. The coordinates of the optimised gate location were $\mathrm{X}=0$, and $\mathrm{Y}=-19.43$.

Table 3. Comparison between FE simulation, ANN and experimental fabrication results.

\begin{tabular}{lc}
\hline & Weld line length $(\mathrm{mm})$ \\
\hline Moldflow simulation & 0.1 \\
ANN prediction & 0.094 \\
Experimental fabrication & 0 \\
\hline
\end{tabular}

\section{CONCLUSIONS}

Finite element, ANN, and GA were linked in order to determine the optimum gate location. The research results revealed that the proposed approach can effectively help engineers to determine optimal gate location and other design elements to achieve competitive advantages of product quality and costs. The efficiency of the method depends on the selection of adequate search area and accuracy of Moldflow simulations and neural network predictions. In this study, the optimal gate location was determined within technical constraints of mould construction. Thus, some part faces could not be considered as search area for detection of optimum gate location. Further researches can be conducted to explore other mould design elements like gate size, gate shape, and coolant systems in order to achieve the desired features. This method can efficiently optimise mould design elements to avoid high costs and time delays associated with problems discovered at the start of production.

\section{ACKNOWLEDGEMENTS}

The authors would like to thanks to Islamic Azad University for their financial assistance and laboratory facilities.

\section{REFERENCES}

[1] Meiabadi MS, Sharifi F. Optimization of Plastic Injection Molding Process by Combination of Artificial Neural Network and Genetic Algorithm. Journal of Optimization in Industrial Engineering. 2013;6:49-54.

[2] Manoraj M, Ansari MNM. Effects of injection moulding process parameters on impact strength of polypropylene-hydroxyapatite biocomposite. Journal of Mechanical Engineering and Sciences. 2017;11:2581-91.

[3] Vatistas G, Lin S, Kwok C. Reverse flow radius in vortex chambers. AIAA Journal. 1986;24:1872. 
[4] Mehat NM, Kamaruddin S. Investigating the effects of injection molding parameters on the mechanical properties of recycled plastic parts using the Taguchi method. Materials and Manufacturing Processes. 2011;26:202-9.

[5] Bharti P, Khan M, Singh H. Six Sigma Approach for Quality Management in Plastic Injection Moulding Process: A Case Study. International Journal of Applied Engineering Research. 2011;6:303-14.

[6] Zhao J, Mayes R, Chen G, Xie H, Chan PS. Effects of process parameters on the micro molding process. Polymer Engineering \& Science. 2003;43:1542-54.

[7] Huang M-C, Tai C-C. The effective factors in the warpage problem of an injection-molded part with a thin shell feature. Journal of Materials Processing Technology. 2001;110:1-9.

[8] Chiang K-T. The optimal process conditions of an injection-molded thermoplastic part with a thin shell feature using grey-fuzzy logic: A case study on machining the PC/ABS cell phone shell. Materials \& Design. 2007;28:1851-60.

[9] Huang C-Y, Chen W-L, Cheng C-M, Pan C-Y. Product quality prognosis in plastic injection moulding. Production Engineering. 2011;5:59-71.

[10] Bociąga E, Jaruga T. Visualization of melt flow lines in injection moulding. journal of Achievements in Materials and Manufacturing Engineering. 2006;18.

[11] Rahman MM, Kadirgama K, Ab Aziz AS. Artificial neural network modeling of grinding of ductile cast iron using water based $\mathrm{SiO} 2$ nanocoolant. International Journal of Automotive and Mechanical Engineering. 2014;9:1649-61.

[12] Sahid NSM, Rahman MM, Kadirgama K. Neural network modeling of grinding parameters of ductile cast iron using minimum quantity lubrication. International Journal of Automotive and Mechanical Engineering. 2015;11:2608-21.

[13] Khan MAR, Rahman MM, Kadirgama K, Bakar RA. Artificial neural network model for material removal rate of TI-15-3 in electrical discharge machining. Energy Education Science and Technology Part A: Energy Science and Research. 2012;29:1025-38.

[14] Khan MAR, Rahman MM, Kadirgama K, Maleque MA, Bakar RA. Artificial intelligence model to predict surface roughness of Ti-15-3 alloy in EDM process. World Academy of Science, Engineering and Technology. 2011;74:198-202.

[15] Yahya MN, Otsuru T, Tomiku R, Okuzono T. A practical system to predict the absorption coefficient, dimension and reverberation time of room using GLCM, DVP and neural network. International Journal of Automotive and Mechanical Engineering. 2013;8:1256-66.

[16] Najiha MS, Rahman MM, Kadirgama K. Experimental investigation and optimization of minimum quantity lubrication for machining of AA6061-t6. International Journal of Automotive and Mechanical Engineering. 2015;11:272237.

[17] Kamal M, Rahman M. An integrated Approach for fatigue life estimation based on continuum mechanics theory and genetic algorithm. International Journal of Automotive and Mechanical Engineering. 2015;11:2756-70.

[18] Chaki S, Ghosal S. A GA-ANN hybrid model for prediction and optimization of $\mathrm{CO} 2$ laser-mig hybrid welding process. International Journal of Automotive and Mechanical Engineering. 2015;11:2458-70.

[19] Spina R. Optimisation of injection moulded parts by using ANN-PSO approach. journal of Achievements in Materials and Manufacturing Engineering. 2006; 15:146-52. 
[20] Kurtaran H, Ozcelik B, Erzurumlu T. Warpage optimization of a bus ceiling lamp base using neural network model and genetic algorithm. Journal of Materials Processing Technology. 2005;169:314-9.

[21] Shen C, Wang L, Li Q. Optimization of injection molding process parameters using combination of artificial neural network and genetic algorithm method. Journal of Materials Processing Technology. 2007;183:412-8.

[22] Ozcelik B, Erzurumlu T. Comparison of the warpage optimization in the plastic injection molding using ANOVA, neural network model and genetic algorithm. Journal of Materials Processing Technology. 2006;171:437-45.

[23] Tutar M, Karakus A. Computational Study of the Effect of Governing Parameters on a Polymer Injection Molding Process for Single-Cavity and Multicavity Mold Systems. Journal of Manufacturing Science and Engineering. 2010;132:011001.

[24] Chen W-L, Huang C-Y, Huang C-Y. Finding efficient frontier of process parameters for plastic injection molding. Journal of Industrial Engineering International. 2013;9:25.

[25] Chen W-C, Kurniawan D. Process parameters optimization for multiple quality characteristics in plastic injection molding using Taguchi method, BPNN, GA, and hybrid PSO-GA. International Journal of Precision Engineering and Manufacturing. 2014;15:1583-93.

[26] Spina R, Walach P, Schild J, Hopmann C. Analysis of lens manufacturing with injection molding. International Journal of Precision Engineering and Manufacturing. 2012;13:2087-95.

[27] Shayfull Z, Shuaib N, Ghazali M, Nasir S, Nooraizedfiza Z. Optimizing length of weld line formation in thin plate by taguchi method and analysis of variance (ANOVA). International Journals of Engineering \& Technology. 2011;11:20771185.

[28] Zhou H, Li D. Computer evaluation of weld lines in injection-molded parts. Journal of reinforced plastics and composites. 2005;24:315-22.

[29] Kim JK, Park SH, Jeon HK. The effect of weld-lines on the morphology and mechanical properties of amorphous polyamide/poly (ethylene-ran-propylene) blend with various amounts of an in situ compatibilizer. Polymer. 2001;42:220921. 